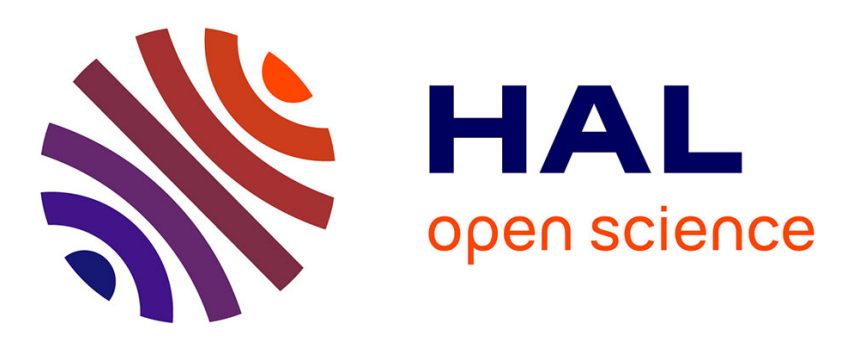

\title{
Flavin-Conjugated Nanobombs: Key Structural Requirements Governing Their Self-Assemblies' Morphologies
}

Jonathan Ribes, Nataliia Beztsinna, Remy Bailly, Sabine Castano, Estelle Rascol, Nada Taib-Maamar, Eduard Badarau, Isabelle Bestel

\section{To cite this version:}

Jonathan Ribes, Nataliia Beztsinna, Remy Bailly, Sabine Castano, Estelle Rascol, et al.. FlavinConjugated Nanobombs: Key Structural Requirements Governing Their Self-Assemblies' Morphologies. Bioconjugate Chemistry, 2021, 32, pp.553 - 562. 10.1021/acs.bioconjchem.1c00028 . hal03448525

\section{HAL Id: hal-03448525 \\ https://hal.science/hal-03448525}

Submitted on 25 Nov 2021

HAL is a multi-disciplinary open access archive for the deposit and dissemination of scientific research documents, whether they are published or not. The documents may come from teaching and research institutions in France or abroad, or from public or private research centers.
L'archive ouverte pluridisciplinaire HAL, est destinée au dépôt et à la diffusion de documents scientifiques de niveau recherche, publiés ou non, émanant des établissements d'enseignement et de recherche français ou étrangers, des laboratoires publics ou privés. 


\title{
Flavin-Conjugated Nanobombs: Key Structural Requirements Governing Their Self-Assemblies' Morphologies
}

\author{
Jonathan Ribes, Nataliia Beztsinna, Remy Bailly, Sabine Castano, Estelle Rascol, Nada Taib-Maamar, \\ Eduard Badarau,* and Isabelle Bestel
}

Cite This: Bioconjugate Chem. 2021, 32, 553-562

Read Online

ACCESS | Lill Metrics \& More | 国 Article Recommendations ｜ st Supporting Information

ABSTRACT: In contrast to artificial molecules, natural photosensitizers have the benefit of excellent toxicity profiles and of life-compatible activating energy ranges. Flavins are such photosensitizers that were selected by nature in a plethora of light-triggered biochemical reactions. Flavin-rich nanoparticles could thus emerge as promising tools in photodynamic therapies and in active-targeting drug delivery. Selfassembled flavin-conjugated phospholipids improve the pharmacokinetics of natural flavins and, in the case of controlled morphologies, reduce photobleaching phenomena. The current article presents a proof of concept for the design of riboflavin-rich nanoparticles of tunable morphology from multilamellar patches to vesicular self-assemblies. Coarse-grained simu-

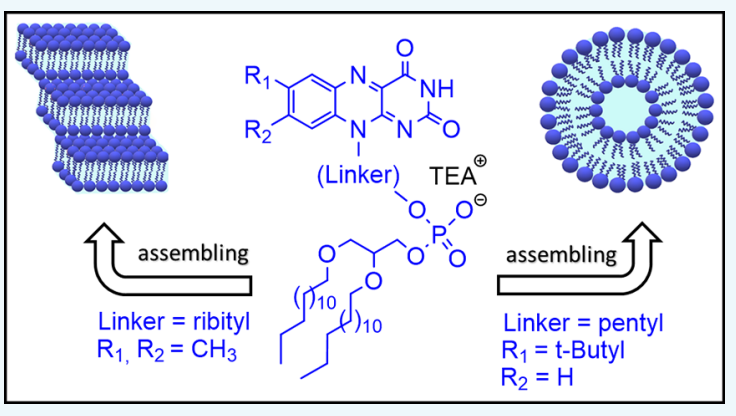
lations of the self-assembling process revealed the key interactions governing the obtained nanomaterials and successfully guided the synthesis of new flavin-conjugates of predictable self-assembly. The obtained flavin-based liposomes had a $65 \mathrm{~nm}$ hydrodynamic diameter, were stable, and showed potential photosensitizer activity.

\section{INTRODUCTION}

Among the various clinically available cancer therapies, photodynamic therapy (PDT) is an emerging technique that has the advantage of affecting only the local environment of stimulated cells without remote damage. PDT relies on the use of light-activated sensitizers, often in the presence of oxygen, to generate local cytotoxic effects. ${ }^{1}$ Despite its many benefits, PDT is not devoid of limitations. Activating the sensitizer by light supposes a highly energetic emission source (typically in the UV range) close to the sensitizer region. Moreover, as for all drugs, the sensitizer itself induces toxicity. This hinders the advantage of the "local-toxicity profile" concept of PDT, as, following a systemic administration, a toxic sensitizer might interfere with other secondary targets or immune system before reaching its primary target. In contrast to artificial sensitizers, natural photosensitizers have an improved toxicity profile. Some of the most promising natural photosensitizers identified to date are the porphyrins and the flavins. Riboflavin (RF, vitamin B2) and its metabolically active derivatives (flavin mononucleotide, FMN; and flavin adenine dinucleotide, FAD) are involved in many light-activated physiological processes., ${ }^{2,3}$ In addition to their biocompatibility advantage, the flavins are easily activated by low-energy blue light $(\lambda=445 \mathrm{~nm})$, contrary to many synthetic photosensitizers which typically require UVA or UVB irradiation. Light-activated riboflavin is already used in a clinical setting either for sterilizing blood samples ${ }^{4}$ or treating keratoconus. ${ }^{5,6}$ In addition to the role of efficient natural photosensitizer, RF can also act as an efficient active agent targeting tumors ${ }^{7}$ because of its native vitamin role: RF transporters were shown to be highly overexpressed in breast $^{8}$ and prostate ${ }^{9}$ cancers as well as in angiogenic endothelium. We showed in the past that, when conjugated on the surface of liposomes, RF was able to target PC3, A431, and HUVEC cells and to enhance liposomal tumoral uptake in mice. $^{10}$

Flavins in general and RF in particular present thus many advantages over other artificial photosensitizers. Unfortunately, the aqueous solubility of RF is low $(\sim 0.1 \mathrm{~g} / \mathrm{L})$ and its elimination half-life is short $\left(t_{1 / 2}=66-88 \mathrm{~min}\right)$ before its renal excretion. One alternative to improve the pharmacokinetics of this photosensitizer might be to develop RF-rich nanoparticles. Many applications of RF-conjugated nanomaterials were described in the literature, ${ }^{11}$ all having either low or uncontrolled amounts of RF in their structure: RF-conjugated dendrimers $^{12,13}$ or polymers, ${ }^{14}$ FMN-conjugated iron-oxides, ${ }^{15}$ or carbon nanotubes ${ }^{16}$ are such examples. The current article presents a proof of concept for the design of tunable flavin-rich

Received: January 19, 2021

Revised: February 15, 2021

Published: February 23, 2021 


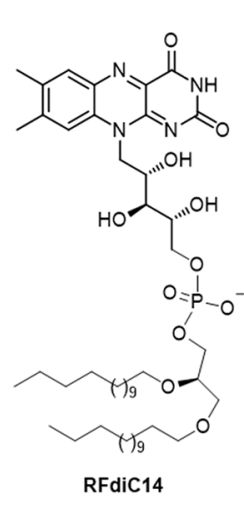

A

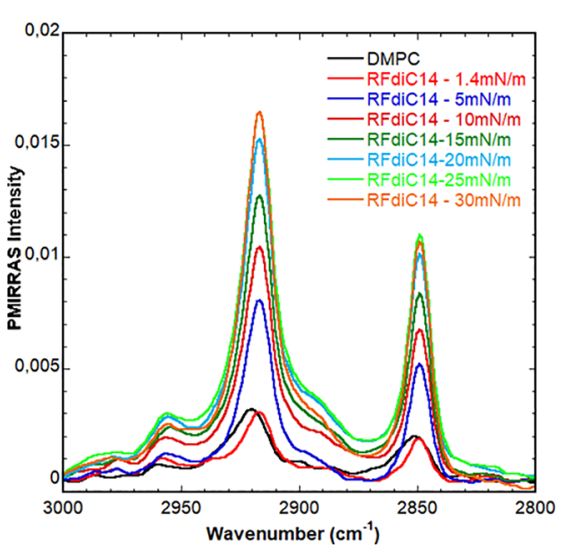

B

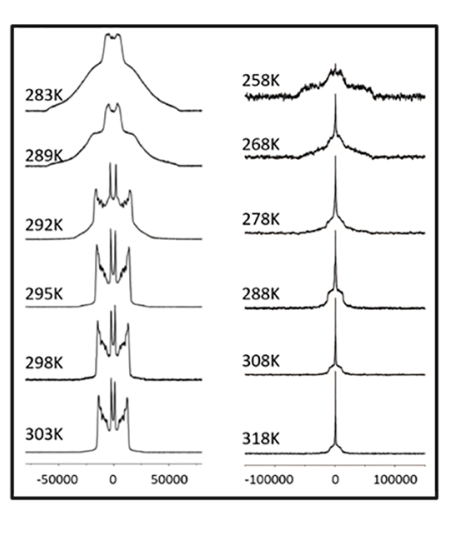

C

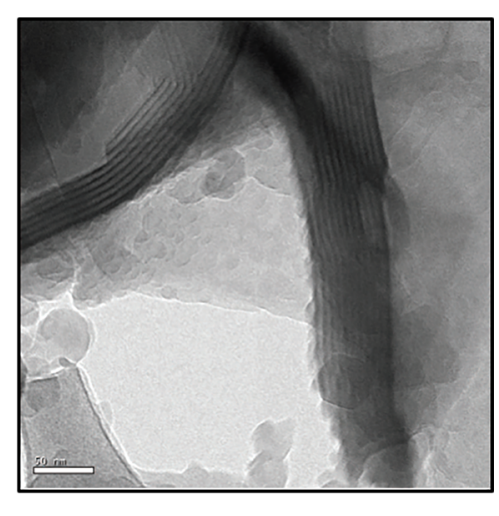

D

Figure 1. (A) Chemical structure of RFdiC14. (B) PMIRRAS spectroscopy profile for RFdiC14. (C) ${ }^{2} \mathrm{H}$ NMR spectra of DMPC-d54 (left) and RFdiC14/DMPC-d54 97.5/2.5 (right). (D) Cryo-EM image of the RFdiC14 self-assembles ( $2 \mathrm{mg}$ lipid/ml HEPES buffer).

nanoparticles incorporating a high (and controlled) ratio of flavins, by combining molecular modeling, organic synthesis, formulation techniques, and physicochemical characterizations. In addition to our primary goal of designing new photosensitizer systems, the same approach could be useful for designing other nanoplatforms for various applications in therapy, optoelectronics, hybrid materials, etc. where the control of the morphology is crucial.

The photosensitizer nanomaterials we target should be obtained starting from phospholipid units via self-assembling processes. We are particularly interested in developing vesicular architectures, because of their ability to deliver drugs to their site of action. This type of morphology would also facilitate the monitoring of these nanoparticles in cells, for example by incorporation of specific dyes. However, conjugating photosensitizers (bulky aromatic derivatives) on phospholipids highly impact the morphology of the generated nanomaterials: the intermolecular interactions between the aromatic moieties will guide the self-assembling process generally to a nonvesicular morphology. Rare examples of vesicular amphiphilic nanomaterials incorporating a high ratio of such artificial phospholipids are described in the literature: porphyrins-conjugated nanomaterials are such exceptions. ${ }^{17-20}$

\section{RESULTS AND DISCUSSION}

We expected to obtain this type of nanoparticles by selfassembling RF-conjugated phospholipids (RF-PL). This type of nanoparticles would also have an enhanced biocompatible profile, because the individual constituents (RF and PL) are both nontoxic natural molecules. We already reported in the past the synthesis of one such RF-PL: ${ }^{10}$ the derivative RFdiC14 represented in Figure 1A. When integrated in the structure of a clinically validated liposome composition (DMPC:DMPG 90:10), ${ }^{21}$ it successfully targeted PC3, A431, and HUVEC cell lines. In order to develop the desired RF-rich nanoparticles, our first hypothesis was to check if the RFdiC14 lipid used alone during the formulation process would induce the formation of vesicular nanostructures. A formulation recipe similar to that previously published ${ }^{10}$ was used. Briefly, a thin layer of RFdiC14 was generated by evaporating a chloroform solution of these phospholipids, followed by hydration with a HEPES buffer (20 mM, pH 7.4). Two different concentrations were investigated: 5 and $2 \mathrm{mg} / \mathrm{mL}$. The obtained suspensions were passed through 400, 200, and $100 \mathrm{~nm}$ filters and submitted to DLS. Unfortunately the observed polydispersity index (PDI) was extremely high $(>0.5)$, indicating the existence of several variable-size populations. Intrigued by the morphology of the obtained self-assemblies, nonfiltered solutions were directly investigated by PMIRRAS spectroscopy. As shown in Figure $1 \mathrm{~B}$, the $\mathrm{CH}$ intensity for the $\mathrm{RFdiC} 14$ at $1.4 \mathrm{mN} / \mathrm{m}$ is the same as for a monolayer of DMPC at 30 $\mathrm{mM} / \mathrm{m}$. The signal for RFdiC14 is more than 5-fold higher at $30 \mathrm{mN} / \mathrm{m}$, indicating a multilamellar aggregation state.

Additional solid-state NMR experiments were subsequently conducted in order to analyze the fatty chain order. In fact, this is related to the mobility of the carbon-deuterium (C-D) bond orientation and can be determined from the $2 \mathrm{H}-\mathrm{NMR}$ quadrupolar splittings. $\mathrm{CD}_{2}$ moieties closer to the glycerol backbone are typically more ordered than terminal $\mathrm{CD}_{2}$ or $\mathrm{CD}_{3}{ }^{22}$ Because RFdiC14 is not ${ }^{2} \mathrm{H}$-labeled, its fatty chain order parameter was investigated by adding a small amount of DMPC-d54 (2.5\% molar) during the formulation step and monitoring its signal by ${ }^{2} \mathrm{H}$ NMR for the obtained aggregates. Pure deuterated DMPC-d54 bilayers were also used as reference during the same experiments. As shown in Figure 1C, the signal of pure DMPC-d54 is characteristic for a lamellar phase: the solid phase signature is observed at $T<298$ $\mathrm{K}$, while at higher temperatures the liquid phase (ld) predominates. In the case of the RFdiC14/DMPC (97.5/ 2.5) mixture, the gel transition temperature was $40{ }^{\circ} \mathrm{C}$ lower: starting at $268 \mathrm{~K}$ and turning completely visible at $258 \mathrm{~K}$. At higher temperatures, the fluid phase is dominant. This proves the fatty chains are highly disordered in the case of RFdiC14, favoring thus a liquid state. Please note the presence of an isotropic peak in the center of the RFdiC14/DMPC-d54 spectra. Integrating for $\sim 10 \%$ of the signal, this is probably specific to the DMPC-d54 small micelle/vesicle formation, which is excluded from the RFdiC14 self-assemblies.

The final proof for the generation of a multibilayer assembly was undoubtedly revealed by cryo-electronic microscopy. Both concentrations ( 5 and $2 \mathrm{mg} / \mathrm{mL}$ ) delivered the same results: variable-size patches of multilamellar morphologies were observed. For example, as represented in Figure 1D, a multilamellar organization in up to 9 layers was generated by self-assembling. The thickness of the layers for the white and the black bands were $2 \pm 0.5$ and $3 \pm 0.5 \mathrm{~nm}$, respectively. The fatty chain region seems thus up to $25 \%$ lower than a standard DMPC bilayer $(2.5 \mathrm{~nm})$. The $5 \pm 0.7 \mathrm{~nm}$ repetition motif 
seemed to suggest that RFdiC14 induced unusual compact bilayers compared to their natural counterparts.

Intuitively, we thought that the interaction between the isoalloxazine moieties were responsible for the membrane packing. In order to confirm such structural insights (e.g., the driving force of the self-assembling process and the molecular interactions), in silico modeling of the aggregation processes was subsequently conducted. We adopted the molecular dynamics approach (md), hoping to generate a model that could explain the RFdiC14 experimental data. If successful, the same approach could be used to predict self-assembling of other PL analogues, designed in silico to favor a specific morphology.

The simulation of biomolecules highly benefits from coarsegrained (CG) molecular dynamics. ${ }^{23-25}$ The CG approach is particularly well-adapted for investigating the aggregation processes, as the size of the simulated system and the time scale necessary to observe a macroscopic property are relatively important. Among the variety of the CG force fields (FF) described in the literature, ${ }^{26}$ we used the Martini $\mathrm{FF}^{27,28}$ because it has proved its efficacy for membrane simulations ${ }^{29}$ and is versatile and widely validated by the scientific community. $^{30}$

In order to generate the CG topology of RFdiC14 phospholipid, we adopted the fine-grained simulation approach. The key step of this procedure is the design of the initial CG mapping from the starting all-atoms molecule. Inspired by both lipids and nucleic acids, as detailed in the Experimental Section and Supporting Information, the CG RFdiC14 mapping was optimized until a good fitting was obtained between the CG and all-atom (AA) trajectories.

In silico conditions (e.g., solute density, box size, pressure setting, etc.) highly impact the morphologies of self-assemblies (e.g. bilayers). As the concentration of the RF-lipid used for the physicochemical characterization was unreasonably diluted to be reproduced in silico, interfacial tensiometry experiments were used to guide the setting of the modeling conditions. Thus, the surface pressure-mean molecular area isotherm was conducted for RFdiC14 (Figure 2), revealing a molecular area of $\sim 20 \AA^{2} /$ molecule. This value is $50 \%$ lower than that of a regular DMPC bilayer ( $40 \AA^{2} /$ molecule), showing again the formation of compact aggregates. In the case of a molecular system composed of 1000 RFdiC14 molecules, this would

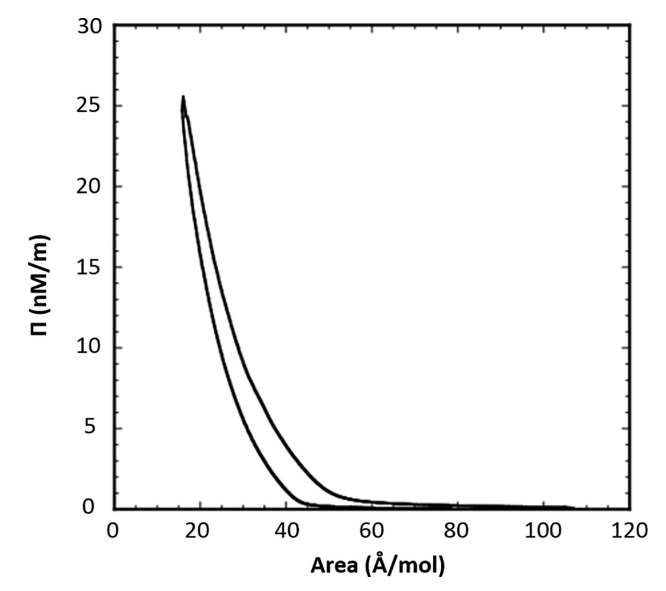

Figure 2. Compression isotherm for $\mathrm{RFdiC} 14$ at the air/buffer interface. Buffer: HEPES $25 \mathrm{mM}, \mathrm{pH} 7.4, \mathrm{~T}=22 \pm 2{ }^{\circ} \mathrm{C}$. Compression speed, $8 \AA^{2} \cdot$ molecule ${ }^{-1} \cdot \mathrm{min}^{-1}$. involve a surface of at least $20 \mathrm{~K} \AA^{2}$. In consequence, we decided to use a reasonably larger box size for our investigated molecular system: a $20 \times 20 \times 20 \mathrm{~nm}$ box size.

Once the key modeling parameters (GC model for RFdiC14, solute density, and box size) were decided, three md replicas were conducted using Gromacs 5.1.4. Briefly, the phospholipid molecules were randomly distributed in the simulation box, and after initial NVT and NPT equilibrations, the simulations were run for $1 \mu \mathrm{s}$ in an isotropic regime, followed by $1 \mu \mathrm{s}$ in a semi-isotropic regime. In all cases RFdiC14 assembled to multibilayers (Figure 3A, center).

Interestingly, the CG riboflavin moieties are not exposed to the water layer but embedded in the bilayer structure, with the apolar grains closer to the fatty core of the membrane, and with the polar grains exposed to the water. In addition, the phosphate, ribityl, and polar isoalloxazine grains are situated in the same water-exposed layer. A complex $\pi$-stacking network among the isoalloxazine moieties could be observed, which is easy to visualize after subsequent backmapping of the CG to the AA resolution (Figure 3A, right). From a physicochemical point of view, it is well-known that this type of organization induces photobleaching of the photosensitizer's activity. It is important to underline that the aromatic cores from one bilayer interact with other isoalloxazine moieties from the vicinal bilayer, which triggers the formation of multilamellar objects as suggested by PMIRRAS.

It is important to mention that the isoalloxazine fragments are also stabilized in the hydrophilic region of the membrane by the polar interaction with the hydroxyl moieties present in the structure of ribityl fragment. The calculated bilayer thickness was $28.5 \AA$. This is $21 \%$ lower compared to the thickness of a DMPC CG model (36 ̊), generated using the same in silico conditions as for RFdiC14 (1000 DMPC molecules $/ 20 \times 20 \times 20 \mathrm{~nm}$ box $)$. While these values are, as expected, higher for CG than for the AA models, the difference between RFdiC14 and DMPC bilayer thicknesses is in agreement with the observed cryo-EM experimental data.

Two key components were thus revealed to induce the multilamellar organization of the RF-conjugated phospholipids: the isoalloxazine and the ribityl fragments. In order to disrupt the lamellar morphology and to potentially generate vesicular morphologies, alteration of these fragments was subsequently considered. Thus, regarding the aromatic core, we hypothesized that additional sterical hindrance induced by a tert-butyl moiety (instead of the methyl groups) could destabilize the $\pi$-stacking interactions. Concerning the ribityl fragment, we decided to investigate the deoxygenated pentyl analogue because of its simplified synthesis and its lack of reactivity during the photodynamic activation. One new phospholipid was thus investigated by molecular dynamics: tBRFdiC14_desoxy (Figure 3B, left). Detailed mappings and topologies of this molecule are described in the Supporting Information.

The CG model of this new molecule was generated following the same protocol as described before (vide supra). The same optimum concentration of the solute, as identified before, was used for the simulations: 1000 molecules in a $20 \times$ $20 \times 20 \mathrm{~nm}$ water box. Interestingly, compared to the multibilayers observed for the RFdiC14, a sharp tendency to form monobilayers was observed for this new phospholipid (Figure 3B, center). As in the case of RFdiC14, the isoalloxazine moiety is embedded in the bilayer structure, with the polar uracil fragment exposed to the outer hydrophilic 
A
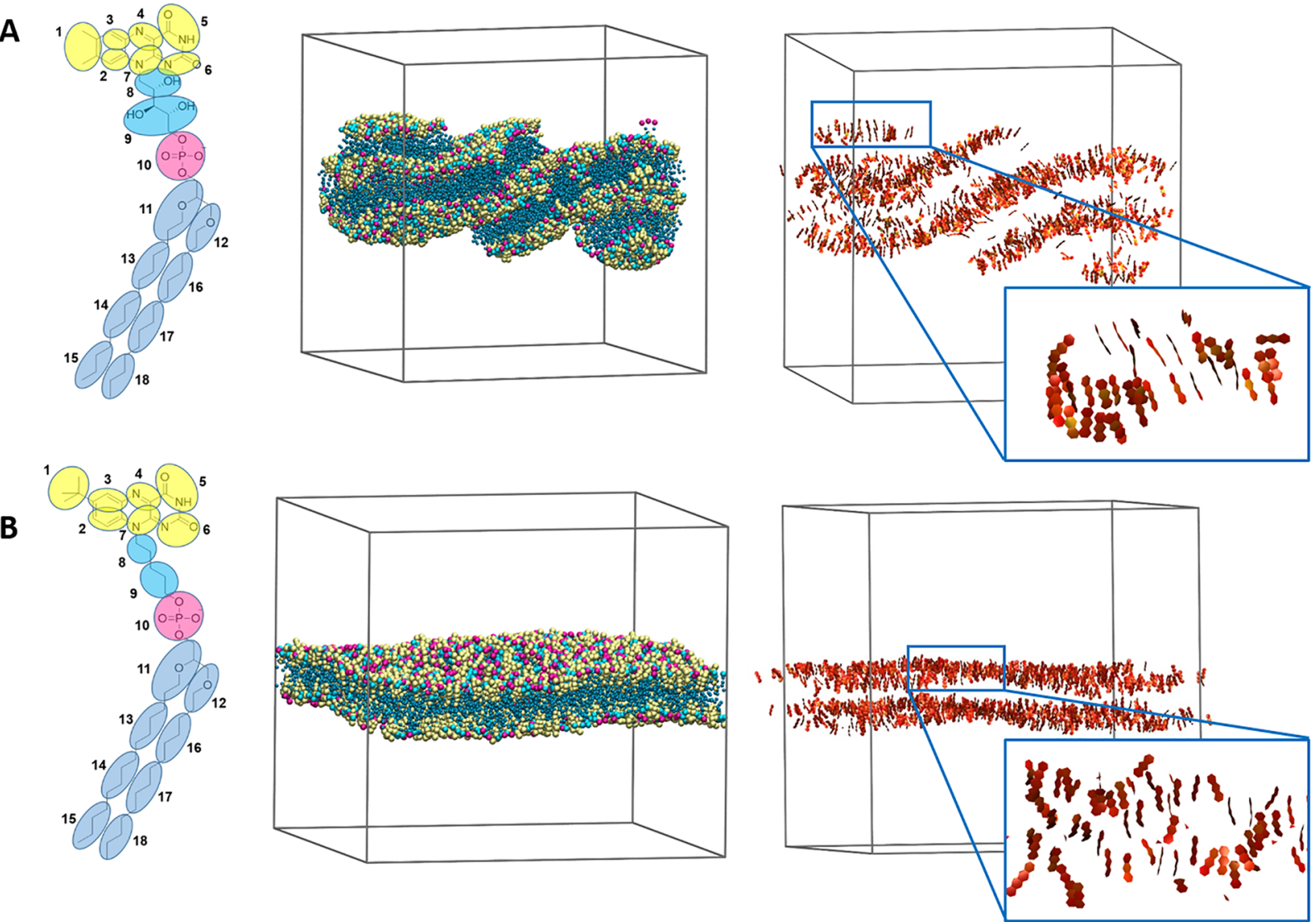

Figure 3. (left) AA-to-CG mappings for RFdiC14 (A) and tBRFdiC14 desoxy (B); (center) CG models for the RFdiC14 (A) and tBRFdiC14 (B) self-assembling obtained after $2 \mu \mathrm{s}$ of dynamics. Water molecules and ions were excluded for clarity. The grains are colored as follows: isoalloxazine in yellow; ribityl/pentyl in light cyan; phosphate in magenta; glycerol and fatty chains in dark cyan. (right) CG-to-AA backmapping for the same self-assemblies including a zoom of a monolayer region. Only isoalloxazine moieties are represented (as "paperchains"). Images were realized with VMD software. ${ }^{31}$

region of the bilayer. The calculated bilayer thickness for the tBRFdiC14_desoxy model was $25.6 \AA$. This value is slightly lower than the value obtained for the RFdiC14 bilayer (28.5 $\AA$ ) and reveals a much compact bilayer structure than the DMPC bilayer model (36 Å). While the CG-RFdiC14 order parameter (calculated versus the bilayer normal) is misleading by the formation of curvy multilayers, in the case of tBRFdiC14_desoxy the order parameter was calculated as 0.218 for the fatty chains. This value is close to 0 (random conformation of CG bonds to the normal bilayer), showing that the fatty tails are highly disordered. When the same (second-rank) order parameter is calculated for the isoalloxazine moiety a value of 0.674 is obtained, showing that the isoalloxazines are better aligned to the bilayer normal than the fatty chains. This is clearly visible for the CG-backmapped to the AA model (Figure 3B, right).

As the CG simulations showed a high preference for the formation of monobilayers in the case of tBRFdiC14_desoxy lipids, we decided to pursue the synthesis of this in silicodesigned phospholipid. The previously reported synthesis of RFdiC $14^{10}$ was based on the commercial availability of the riboflavin as starting material. However, in the case of tBRFdiC14_desoxy, as the isoalloxazine fragment is not commercially available, the access to this new phospholipid was more challenging.

The synthesis started by the alkylation of the commercial $t$ butylaniline by 5-chloropentanol under refluxing triethylamine.
The alkylated derivative 1 was subsequently reacted with 6chlorouracil. When conducted in a water/dioxane mixture the substitution reaction was particularly slow, delivering less than $40 \%$ of the desired product after refluxing for $24 \mathrm{~h}$. Higher yields $(64 \%)$ were obtained under solvent-free conditions: the starting material and the 6-chlorouracil were mixed under heating at $180{ }^{\circ} \mathrm{C}$ for $30 \mathrm{~min}$ in a sealed tube. The isoalloxazine ring was generated following the two-step procedure originally described by Yoneda: ${ }^{32}$ a first $N$-oxide intermediate was obtained by nitrosation of compound 2 followed by spontaneous cyclization.

The crude product was subsequently reduced by dithiothreitol (DTT) under refluxing ethanol and delivered the desired 7-t-butyl-isoalloxazine 3 in a good yield ( $88 \%$ over two steps). We intended to generate the phosphate moiety from the commercially available 2-cyanoethyl- $N, N$-diisopropylchloro-phosphoramidite (CCPA), a reagent typically used in the oligonucleotide chemistry. On the basis of our previous synthesis of RFdiC14 lipid, ${ }^{10}$ we initially tested the coupling of the CCPA reagent with the intermediate 3 . This step delivered the desired substitution compound; however, contrary to RFdiC14 synthesis, this intermediate spontaneously oxidized to the corresponding phosphoramidate, as indicated by the ${ }^{31} \mathrm{P}$ NMR. This seems to be promoted by the isoalloxazine moiety, as already reported in the literature. ${ }^{33}$ We managed to resolve this synthetic issue by first introducing the phosphorus on the glycerol core, followed by reaction with the derivative 3 . The 
Scheme 1. Reagents and Conditions ${ }^{a}$<smiles>CC(C)(C)c1ccc(NCCCO)cc1</smiles>

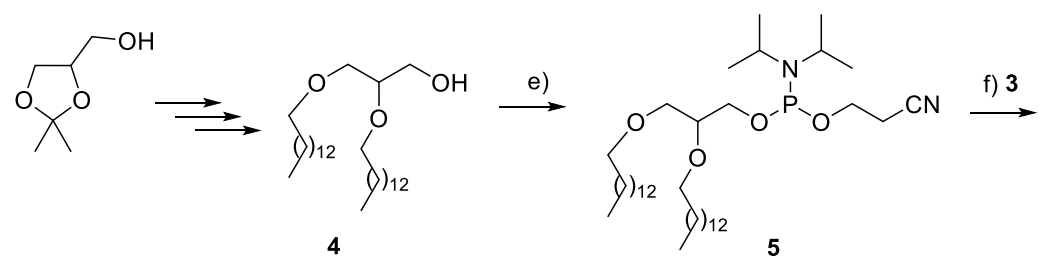

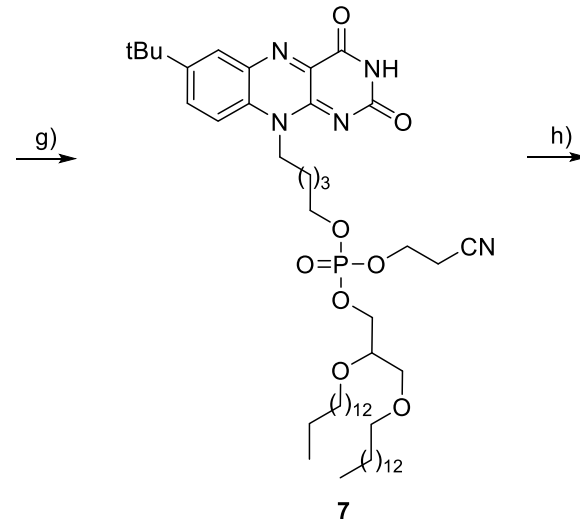

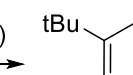<smiles>O=c1cc(N(CCO)c2ccccc2)[nH]c(=O)[nH]1</smiles>

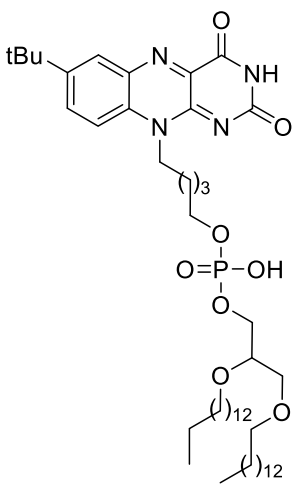

tBRFdiC14_desoxy (8)<smiles>CC(C)(C)c1ccc2c(c1)nc1c(=O)[nH]c(=O)nc-1n2CCCO</smiles><smiles></smiles>

a (a) 5-Chloropentanol, TEA, reflux, $20 \mathrm{~h}$ (69\%); (b) 6-Cl-uracile, sealed tube, $180^{\circ} \mathrm{C}, 30 \mathrm{~min}$ (64\%); (c) $\mathrm{NaNO}_{2}$, acetic acid, r.t., $3 \mathrm{~h}$, then $\mathrm{H}_{2} \mathrm{O}$, r.t., $3 \mathrm{~h}$; (d) DTT, EtOH, reflux, 4 h (88\%, over 2 steps); (e) 2-cyanoethyl- $N, N$-diisopropylchlorophosphoramidite, dry DIPEA, dry DCM, 1 h 30 min, r.t.; (f) BTT, dry THF, r.t., 1 h 30 min; (g) $\mathrm{I}_{2}$ in THF $/ \mathrm{H}_{2} \mathrm{O} /$ pyridine, r.t., 12 h; (h) $\mathrm{MeONa}$, dry $\mathrm{MeOH}$, r.t., 24 h (24\%, over 4 steps).

synthesis of the alkylated glycerol was conducted as reported earlier by our group. ${ }^{10}$ Briefly, starting from the commercially available solketal, the hydroxyl moiety was protected as a $p$ methoxyphenol (PMP) and the isopropylidene was acidhydrolyzed. $^{34}$ The subsequent alkylation of the hydroxyl moieties by 1-bromotetradecane was conducted in dry dimethylformamide under anionic conditions. PMP removal by cerium ammonium nitrate (CAN) in an acetonitrile/water $(8 / 1)$ mixture delivered the alcohol 4 in good yield $(67 \%)$. This was reacted with CCPA, in dry dichloromethane and using diisopropylamine as proton scavenger. Crude 5 was activated by acidic BTT (5-benzylthio- $1 \mathrm{H}$-tetrazole) in acetonitrile and reacted with the isoalloxazine 3 to conduct to the phosphite 6 . Subsequent oxidation of $\mathrm{P}(\mathrm{III})$ to $\mathrm{P}(\mathrm{V})$ was smoothly promoted by iodine in a tetrahydrofuran/water/ pyridine mixture and delivered the phosphate 7. A last step involving the cyanoethyl cleavage under basic conditions $(\mathrm{MeONa} / \mathrm{MeOH})$ resulted in the desired lipid tBRFdiC14_desoxy 8 (Scheme 1). The calculated yield over the last four steps was $24 \%$ ( $70 \%$ yield for each step). It should be mentioned that all the steps involving phosphorus-containing derivatives were monitored by ${ }^{31} \mathrm{P} \mathrm{NMR}$, which proved essential for optimizing the synthetic sequence. Because the synthesis of phospholipid tBRFdiC14_desoxy was successful, we were intrigued if this lipid would promote vesicular nanoparticles.
The same lipid concentration as for RFdiC14 was used. Thus, hydration of a chloroform film of tBRFdiC14_desoxy by a HEPES buffer ( $2 \mathrm{mg}$ lipid/ml buffer) delivered a suspension that was consecutively extruded over 400,200 , and finally 100 $\mathrm{nm}$ filters. The final filtrate was investigated by DLS (Figure 4A) which showed a homogeneous suspension of nanoparticles with a hydrodynamic diameter of $\sim 65 \mathrm{~nm}$. Nanoparticle tracking analysis (NTA) confirmed a similar size $(74 \mathrm{~nm})$ of the nanoparticles. Rewardingly, the cryo-EM analysis of the same suspension proved the formation of vesicular morphologies specific to liposomes (Figure 4C).

The stability of tBRFdiC14_desoxy-induced liposomes was monitored by DLS over a period of 12 days at 4 and $20^{\circ} \mathrm{C}$. The clinically validated liposome mixture (DMPC/DMPG 9/ 1) was also tested during the same experiment as reference. As shown in Figure 4B, the samples incorporating the flavinconjugated nanoparticles were stable during the tested period and showed an average hydrodynamic diameter of $62 \mathrm{~nm}( \pm 5$ $\mathrm{nm})$ at $4{ }^{\circ} \mathrm{C}$ and $67 \mathrm{~nm}( \pm 8 \mathrm{~nm})$ at $20{ }^{\circ} \mathrm{C}$.

The ability of the flavin-conjugated liposomes to act as potential photosensitizers was subsequently evaluated using the protocol originally described by Chen et al. ${ }^{35}$ and which was further optimized. As detailed in the Experimental Section, a low-energy blue LED can be used to activate flavins to their reactive triplet state that can promote type I or II radical reactions. ${ }^{36}$ The ability of the light-activated liposomes to induce reduction of the colorless MTT to the corresponding 
A

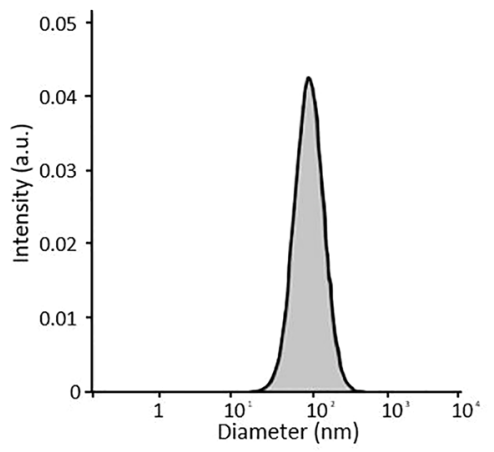

C

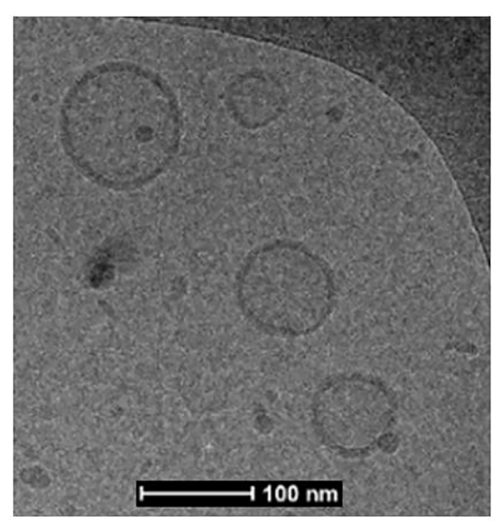

B

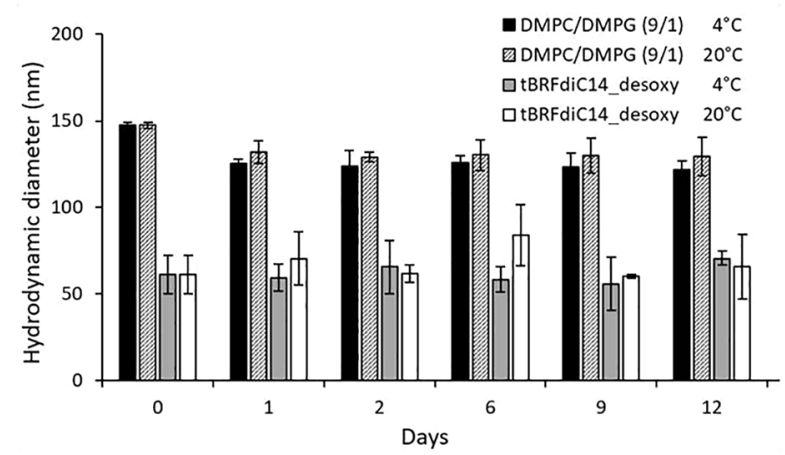

D

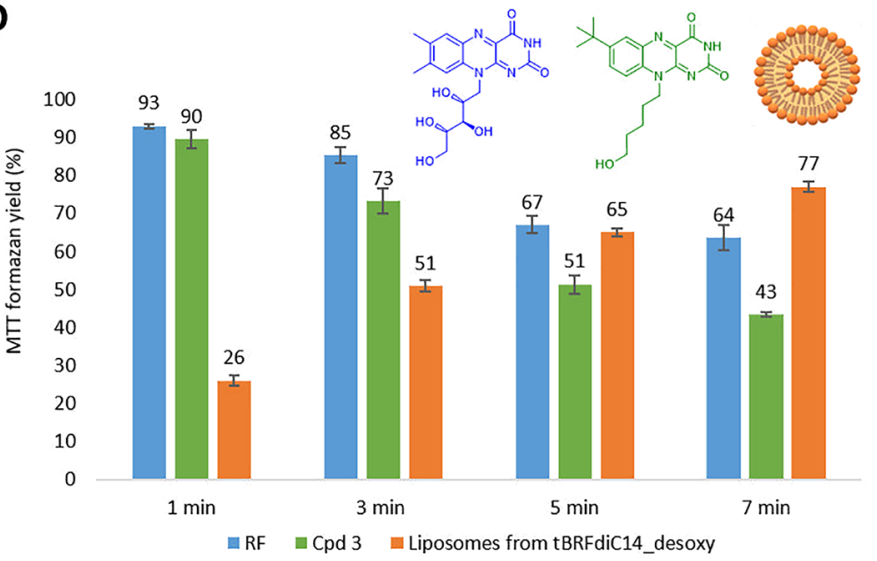

Figure 4. (A) DLS profile for the tBRFdiC14_desoxy self-assemblies (2 mg lipid/ml HEPES buffer). (B) Stability of tBRFdiC14_desoxy-induced liposomes over 12 days. (C) Cryo-EM image of the same nanoparticles. (D) Photodynamic activity of flavin-based photosensitizers, expressed as the conversion (\%) of MTT to MTT formazan (experiments were run in triplicate, SD $\pm 3 \%$ ).

blue formazan was compared to that of the isolated flavins ( $R F$ and $\mathrm{tBdRF}-\mathrm{cpd} 3)$. As shown in Figure 4D, irradiation with a $96 \mathrm{~mW}$ blue LED $\left(\lambda_{\mathrm{em}}=440 \mathrm{~nm}\right)$ of the same flavinconcentration samples showed a time-dependent activity for all the investigated flavin species. Interestingly, if the solutionflavins and the generated MTT formazan were less stable over time (as indicated by the decrease of the MTT formazan yield from $90 \%$ to $43 \%$ for cpd 3 ), in the case of the formulatedflavins the yield is constantly increasing from $26 \%$ to $77 \%$ from 1 to $7 \mathrm{~min}$, respectively. This seems to suggest that flavindoped liposomes have a strong potential as stable and efficient photosensitizers and that the morphology plays a crucial role in regulating flavin reactivity.

In addition to their promising photosensitizer activity, in contrast to solution-flavins which are limited by their low water solubility and their fast elimination from cells, the flavin-doped liposomes would have the advantage of a preferable accumulation in tumors (EPR effect), increasing their concentration beyond the physiologically relevant concentrations of flavins $(0.01 \mu \mathrm{M}$ for RF, $0.006 \mu \mathrm{M}$ for FMN $){ }^{37}$ By blue light activation, their local cytotoxic effect should thus be greatly enhanced compared to natural flavins.

Additional studies are currently being conducted in our laboratory to also prove their ability to efficiently transport various cargo in a biological environment. The high ratio of flavin present in their polar region makes them particularly well-adapted to transport redox-sensitive species, as flavins can act as redox scavengers.

Several other flavin-based conjugates are currently synthesized by our group in order to further potentiate the photosensitizer activity of such "nanobombs", which could be finally used as a viable PDT solution for clinical applications.

\section{CONCLUSIONS}

The current article reports a new generation of amphiphilic nanomaterials obtained from flavin-conjugated phospholipids (PL). When RF was inserted in the polar region of a natural phospholipid, the aqueous-promoted self-assembly had a multilamellar morphology. A coarse-grained (CG) molecular model was successfully developed for this flavin-derived PL in order to reveal the intermolecular details that governed the self-assembling process. Despite a constantly increasing interest in flavins, due to their redox, fluorescent, and photosensitive properties, to the best of our knowledge, no such CG models were reported for flavins and their derivatives in the literature. The CG models we designed are available in the Supporting Information and/or on demand. These CG models could be used to simulate other macroscopic properties of flavins, or it can be easily adapted to other flavin analogues. This modeling approach was used to validate in silico a new flavin conjugate, designed to induce vesicular self-assemblies. Rewardingly, the successful synthesis of this new flavinconjugated PL, carrying a bulky ter-butyl moiety in the isoalloxazine region, allowed subsequent generation of liposomes of $\sim 65 \mathrm{~nm}$ (by DLS, NTA, and cryo-TEM). Rare cases of such liposomes, generated only from PL having bulky aromatic moieties in their structure, were described before in the literature; porphysomes (liposomes from porphyrinconjugated PL) are notable examples of this type.

The vesicular flavin-doped nanoparticles could be exploited in anticancer drug delivery via the EPR effect alone, or in 
synergy with the active targeting, using a flavin-based active targeting agent during the formulation step, as we showed in the past. ${ }^{10}$ The flavin-doped liposomes have a promising photosensitizing potential. Additional biological studies will confirm if their light-triggered cytotoxicity could be exploited in antitumor therapy in order to validate the concept presented herein.

\section{EXPERIMENTAL SECTION}

Molecular Modeling. Molecular dynamics calculations were conducted using Gromacs 5.1.4 software $^{38}$ and the Martini force fields (martini_v2.2, martini_v2.0_ions and martini_v2.0_lipids) on a $7 \overline{2} \mathrm{CPUs}$ (@3.3 GHz) $/ 64$ GB RAM server. Unified all-atoms topologies for both lipids were generated by the ATB server (RFdiC14: Molid 363434 and tBRFdiC14_desoxy: Molid 362814).

CG models for both lipids were generated using the allatoms (AA) simulation approach. Briefly, molecular systems were prepared from 128 lipids in a $6 \times 6 \times 12 \mathrm{~nm}$ box, hydrated and neutralized with $\mathrm{NaCl}$, then equilibrated in the constant-NVT ensemble for 2 ns and subsequently in the constant-NPT ensemble for an additional $2 \mathrm{~ns}$ at $320 \mathrm{~K}$ and 1 bar. A first MD simulation ( $2 \mathrm{~ns}$, isotropic pressure coupling using the Parrinello-Rahman barostat) ${ }^{39}$ was conducted for the AA lipids in water. Subsequently, the fine-grained trajectory was converted to a "mapped" coarse-grain trajectory by assigning the center-of-mass (COM) of the atoms to the corresponding "mapped" CG beads (M_CG). Based on the initial CG topology, a second simulation (2 ns, isotropic pressure coupling using the Parrinello-Rahman barostat) was conducted, this time for the coarse-grained RFdiC14 in water. The distribution functions for the bonds (B), angles (A), and dihedrals (D) for this "real" CG (R CG) trajectory were calculated and compared with the distributions functions of the M_CG trajectory. The coarse-grain parameters (B, A, D, and force constants) were repeatedly optimized until both distributions reasonably overlapped, indicating that the R_CG trajectory simulated well the M_CG trajectory (obtained from AA MD).

The generation of the CG topologies allowed the subsequent simulations of the self-assembling processes. For each lipid, 1000 molecules were randomly distributed in a cubic box of $20 \times 20 \times 20 \mathrm{~nm}$. The system was hydrated, neutralized, and subsequently equilibrated in the constant$N V T$ ensemble for $10 \mathrm{~ns}$ then in the constant-NPT ensemble for $20 \mathrm{~ns}$. Temperature and pressure were maintained constant at $320 \mathrm{~K}$ and $1 \mathrm{bar}$, respectively, using the Berendsen thermostat and barostat. ${ }^{40}$ Three to five MD replicas were performed for each lipid with a $20 \mathrm{fs}$ time step, a temperature of $320 \mathrm{~K}$ set using the Bussi et al. velocity rescaling thermostat comprising an initial $1 \mu \mathrm{s}$ trajectory at the isotropic pressure, followed by at least $1 \mu \mathrm{s}$ at the semi-isotropic pressure of $1 \mathrm{bar}$ maintained with a Parrinello-Rahman barostat.

CG backmapping to AA was carried out using the "backward.py" script developed by Wassenaar et al., ${ }^{41}$ while the calculation of the fatty chains order parameter was done with the "do-order-gmx5.py" script manually modified to integrate the flavin-lipids. Both tools were downloaded from the Martini web site. ${ }^{30}$ Bilayer thickness was calculated as the distance between the $\mathrm{PO}_{4}$ density peaks of the bilayers (calculated with the gmx density tool from Gromacs 5.1.4). The order parameter for tBRFdiC14_desoxy lipid was calculated for the last $100 \mathrm{~ns}$ of the $\overline{1} \mu \mathrm{s}$ semi-isotropic trajectories, for the following "CG-bonds": 1-3, 3-4, 4-5, 27 , and $7-6$ for the isoalloxazine moiety and 12-16, 16-17, $17-18,11-13,13-14$, and $14-15$ for the fatty chains (CG numbering is indicated in Figure 3B).

Formulation. Self-assemblies were prepared using the standard film hydration method. ${ }^{42}$ RFdiC14, tBRFdiC14 desoxy, and DMPC/DMPG (90:10) phospholipids (1 mg) were dissolved in chloroform $(1 \mathrm{~mL})$ in a round-bottom flask, and the solvent was slowly evaporated under nitrogen. The flask was subsequently dried under vacuum overnight to remove the residual traces of solvent. The obtained phospholipid layer was subsequently hydrated with $0.5 \mathrm{~mL}$ of $0.25 \mathrm{mM}$ HEPES buffer ( $\mathrm{pH} 7.4$ ) to a final lipids concentration of $2 \mathrm{mg} / \mathrm{mL}$. Hydrated films were then subjected to 5 freeze-thaw cycles with the help of liquid nitrogen and a water bath $\left(50{ }^{\circ} \mathrm{C}\right)$. The obtained self-assemblies were extruded 21 times through a $100 \mathrm{~nm}$ polycarbonate membrane (Avanti Polar Lipids, United States).

Spectroscopy. PMIRRAS. The spectra were recorded on a Nicolet Nexus 870 spectrometer equipped with a photovoltaic $\mathrm{HgCdTe}$ detector cooled at $77 \mathrm{~K}$. Spectra were obtained by performing 800 scans at a resolution of $8 \mathrm{~cm}^{-1}$. Briefly, PMIRRAS combines FT-IR reflection spectroscopy with fast polarization modulation of the incident beam between parallel (p) and perpendicular (s) polarizations. The differential reflectivity spectrum is obtained using a two-channel processing signal. To remove the contribution of liquid water absorption and the dependence on Bessel functions, the monolayer spectra are divided by that of the subphase. With an incidence angle of $75^{\circ}$, transition moments preferentially oriented in the plane of the interface give intense and upward oriented bands, while perpendicular ones give weaker and downward oriented bands. ${ }^{43}$

Solid-State NMR. Sample Preparation. Three sets of samples were prepared: DMPC:DMPG (90:10), DMPC:DMPG:RfdiC14 (90:10:10), and RfdiC14:DMPC $(100: 2,5)$ with the total lipid concentration of $20 \mathrm{mM}$. For all samples DMPC-d54 (Avanti Polar Lipids, United States) with fully deuterated acyl chains was used. Lipid mixtures were dissolved in chloroform, and the solvent was evaporated under gentle nitrogen stream. The obtained lipid films were taken up in $500 \mu \mathrm{L}$ of Milli-Q water, hydrated at $45{ }^{\circ} \mathrm{C}$ for $30 \mathrm{~min}$, and then subjected to 5 freeze-thaw cycles. They were subsequently frozen in liquid nitrogen and lyophilized (DuraDryMP from FTS Systems). The obtained powder was taken up in $100 \mu \mathrm{L}$ of light water (containing 2-3 ppm of deuterium, CortectNet, France) for ss-NMR studies. Three additional freeze-thaw cycles were conducted $\left(10 \mathrm{~min}\right.$ at $45^{\circ} \mathrm{C} /$ stirring/ freezing in liquid nitrogen) before the samples were transferred to $4 \mathrm{~mm}$ zircon oxide rotors (CortectNet, France).

NMR Experiment. The NMR spectrometer RMN $800 \mathrm{MHz}$ SB Bruker (Wissembourg, France) equipped with the probe CP-MAS SB 1H/BB able to rotate at the magic angle was used for the solid NMR studies. The echo solid sequence $\left(90^{\circ}-\tau-\right.$ $90^{\circ}-\tau$-Acq) was employed for the deuterium observations. The pulses at $90^{\circ}$ were $2.88 \mu \mathrm{s}$; the echo delays $(\tau)$ were $40 \mu \mathrm{s}$ with the registering from $4 \mathrm{k}$ points. The repeating time was $2 \mathrm{~s}$ with the acquisition number from 1 to $12 \mathrm{k}$ according to the sample. The temperature was varied from 20 to $50{ }^{\circ} \mathrm{C} \pm 5{ }^{\circ} \mathrm{C}$. The obtained signals were filtered with a decreasing exponential function of the constant between 100 and 500 $\mathrm{Hz}$ and then subjected to Fourier transformation from the top of the echo spin. Finally, the data were analyzed with Topspin 3.2 software (Bruker, United States). 
Physico-Chemical Analysis. Surface Pressure Measurements. Monolayer experiments were performed with a computer-controlled Langmuir film balance (Nima Technology, Coventry, England). The rectangular trough and the barrier were made of Teflon. The surface pressure $(\pi)$ was measured by the Wilhelmy method using a filter paper plate. The trough was filled with ultra pure water (Milli-Q Millipore). The experiments were carried out at $22 \pm 2{ }^{\circ} \mathrm{C}$. RFdiC14 films were obtained by spreading a few microliters of RFdiC14 stock solution $\left(1.22 \mathrm{mM}\right.$ in $\left.\mathrm{CHCl}_{3}\right)$ at the air/buffer (HEPES $25 \mathrm{mM}, \mathrm{pH}$ 7.4) interface. The solvent was evaporated over $10 \mathrm{~min}$, and the obtained lipid film was slowly compressed and decompressed at the same speed a during the compression $\left(8 \AA^{2} \cdot\right.$ molecule $\left.^{-1} \cdot \mathrm{min}^{-1}\right)$.

$D L S$. Size and distribution measurements of control and specific liposomes were performed in triplicate by dynamic light scattering (DLS) using a Vasco Nanoparticle Size Analyzer (Cordouan Technologies, France) at $25{ }^{\circ} \mathrm{C}$. Calculations of size distribution based on light-scattering intensity were performed with NanoQ software version 2.5.9.0. The liposomal stock dispersions were diluted by a factor of 15 with a 25 or $10 \mathrm{mM}$ HEPES buffer before measurements were performed by DLS.

NTA. Size, distribution, and concentration measurements of control and specific liposomes were performed in triplicate by nanoparticle tracking analysis (NTA) using a Nanosight NS300 NTA (Malvern) at $25{ }^{\circ} \mathrm{C}$. Calculations of the size were based on the direct conversion of the tracks by finite track length adjustment and were processed with NTA $3.2 \mathrm{Dev}$ Build 3.2.16. The liposomal stock dispersions $(2 \mathrm{mg} / \mathrm{mL})$ were diluted by a factor of 10000 with a $10 \mathrm{mM}$ HEPES buffer $\mathrm{pH}$ 7.4 before measurements.

Cryo-EM. The RfdiC14 and tBRFdiC14_desoxy autoassemblies were prepared with the same method as described earlier for the liposome preparation. Two concentrations of $\mathrm{RfdiC} 14$ were used ( 2 and $5 \mathrm{mg} / \mathrm{mL}$ ) and one concentration for tBRFdiC14_desoxy $(2 \mathrm{mg} / \mathrm{mL})$. In the case of RFdiC14, nonextruded samples were investigated, whereas for tBRFdiC14, both nonextruded and extruded samples were investigated. Cryo-EM analysis was performed as described previously. ${ }^{10}$ For the sample preparation, $5 \mu \mathrm{L}$ of sample aliquot was deposited onto an EM grid coated with a perforated carbon film (Ted Pella, Redding, CA, United States); the excess liquid was blotted off with a filter paper; and the grid was then quickly plunged into liquid ethane using a Leica EM-CPC cryo-chamber. EM grids were stored in cryoboxes under liquid nitrogen until use and then mounted in a Gatan 626 cryo-holder and transferred in a Tecnai F20 microscope operated at $200 \mathrm{kV}$. Images were recorded with an USC1000-SSCCD Gatan camera (for RFdiC14) or with an FEI Eagle CCD camera (tBRFdiC14_desoxy). The size of the objects was measured with Image $\mathrm{v} 1.51$ software. ${ }^{44}$

Photosensing Activity. Stock aqueous solutions of riboflavin $\left(1.275 \times 10^{-4} \mathrm{M}, \mathrm{pH} 6.5\right)$, $t$-butyl desoxyriboflavin $\left(1.275 \times 10^{-4} \mathrm{M}, \mathrm{pH} 6.5\right)$, thiazolyl blue tetrazolium bromide $\left(\mathrm{MTT}, 1.19 \times 10^{-5} \mathrm{M}\right)$, and L-methionine $\left(5.66 \times 10^{-2} \mathrm{M}\right)$ were freshly prepared using ultrapure water before the experiments. The liposomes were prepared as described before, and their size was checked by DLS. Flavin content in liposomes was determined via a calibration curve of tBRFdiC14_desoxy in $\mathrm{MeOH}$ obtained using a freshly prepared stock solution of tBRFdiC14_desoxy in methanol $(1.11 \mathrm{mM})$ and analyzed by a Jasco V-760 spectrophotometer
(SpectraManager software). Eight acquisitions were realized at a wavelength of $\lambda=442 \mathrm{~nm}$ for different concentrations between 0 and $1.54 \times 10^{-4} \mathrm{M}\left(y=0.992 x-0.004, R^{2}=\right.$ 0.9989). A dilution step of liposome solution in water is required to adjust the concentration to $1.275 \times 10^{-4} \mathrm{M}$.

Riboflavin, tert-butyl desoxyriboflavin, or liposomes solutions $\left(0.444 \mathrm{~mL}, 5.66 \times 10^{-5} \mathrm{mmol}\right)$ were mixed with 0.056 $\mathrm{mL}$ of the second solution (MTT, $5.27 \times 10^{-5} \mathrm{mmol}$; and $\mathrm{L}-$ methionine, $3.175 \times 10^{-3} \mathrm{mmol}$ ) in a standard test tube (Fisher Scientific ref. 11555914) under air atmosphere. The reaction mixture was irradiated by direct contact between the reaction vial and a blue $\operatorname{LED}\left(\lambda_{\mathrm{em}}=455 \mathrm{~nm}\right)$ at $96 \mathrm{~mW}$ (M455F3 LED, optical fiber M28L01 $0.4 \mathrm{~mm}$, and cube driver LEDD1B were purchased from Thorlabs). The output LED light intensity was measured using the Thorlab PM100D Console and a S120VC probe prior to irradiation experiments. The reaction mixture was irradiated for $1,3,5$, and $7 \mathrm{~min}$. The generated suspension of MTT formazan was solubilized by adding THF $(0.5 \mathrm{~mL})$. Its absorbance was measured at $\lambda=560$ $\mathrm{nm}$ and reported to a calibration curve prepared with commercial MTT formazan in $\mathrm{THF} / \mathrm{H}_{2} \mathrm{O}(1 / 1)$ for concentrations between 0 and $5.34 \times 10^{-5} \mathrm{M}(y=0.0631 x$ $\left.-0.0152, R^{2}=0.9991\right)$.

\section{ASSOCIATED CONTENT}

\section{SI Supporting Information}

The Supporting Information is available free of charge at https://pubs.acs.org/doi/10.1021/acs.bioconjchem.1c00028.

Synthesis details, characterization data for new compounds, ${ }^{1} \mathrm{H}$ and ${ }^{13} \mathrm{C}$ NMR spectra, flavin mapping, and topologies (PDF)

\section{AUTHOR INFORMATION}

\section{Corresponding Author}

Eduard Badarau - University of Bordeaux, CNRS, CBMN, UMR 5248, Institute of Chemistry and Biology of Membranes \& Nano-objects (CBMN), 33600 Pessac, France; (1) orcid.org/0000-0002-0460-2338;

Email: edouard.badarau@u-bordeaux.fr

\section{Authors}

Jonathan Ribes - University of Bordeaux, CNRS, CBMN, UMR 5248, Institute of Chemistry and Biology of Membranes \& Nano-objects (CBMN), 33600 Pessac, France

Nataliia Beztsinna - University of Bordeaux, CNRS, CBMN, UMR 5248, Institute of Chemistry and Biology of Membranes \& Nano-objects (CBMN), 33600 Pessac, France

Remy Bailly - University of Bordeaux, CNRS, CBMN, UMR 5248, Institute of Chemistry and Biology of Membranes \& Nano-objects (CBMN), 33600 Pessac, France

Sabine Castano - University of Bordeaux, CNRS, CBMN, UMR 5248, Institute of Chemistry and Biology of Membranes \& Nano-objects (CBMN), 33600 Pessac, France

Estelle Rascol - University of Bordeaux, CNRS, CBMN, UMR 5248, Institute of Chemistry and Biology of Membranes \& Nano-objects (CBMN), 33600 Pessac, France

Nada Taib-Maamar - University of Bordeaux, CNRS, CBMN, UMR 5248, Institute of Chemistry and Biology of Membranes \& Nano-objects (CBMN), 33600 Pessac, France

Isabelle Bestel - University of Bordeaux, CNRS, CBMN, UMR 5248, Institute of Chemistry and Biology of Membranes \& Nano-objects (CBMN), 33600 Pessac, France 
Complete contact information is available at:

https://pubs.acs.org/10.1021/acs.bioconjchem.1c00028

\section{Notes}

The authors declare no competing financial interest.

\section{ACKNOWLEDGMENTS}

The authors acknowledge Dr. Erick J. Dufourc and Axelle Grélard for their support in conducting the ss-NMR experiments. Support from the cryo-TEM platform (Sisareuth Tan and Prof. Alain Brisson) at CBMN is also acknowledged. Authors acknowledge Dr. Marie Morille and Pr. Joël Chopineau at ICGM (UMR 5253) for access to Nanosight NS300 and support in experiments. This project was financially supported by the "Ligue Contre le Cancer" Foundation (Nano-RF/2017 to I.B. and Nano-Bombs/2019 to E.B.).

\section{ABBREVIATIONS}

PDT, photodynamic therapy; FMN, flavin mononucleotide; $\mathrm{FAD}$, flavin adenine dinucleotide; UV, ultraviolet; RF, riboflavin; PC3, human prostate cancer cell line; A431, human epidermoid carcinoma cell line; HUVEC, human umbilical vein endothelial cells; DMPC, 1,2-dimyristoyl-snglycero-3-phosphocholine; DMPG, 1,2-Dimyristoyl-sn-glycero3-phosphoglycerol; HEPES, N-(2-hydroxyethyl)piperazine- $\mathrm{N}^{\prime}$ (2-ethanesulfonic acid); ACN, acetonitrile; CAN, ceric ammonium nitrate; DEAD, diethyl azodicarboxylate; DCM, dichloromethane; DIPEA, diisopropylethylamine; DMF, dimethylformamide; DTT, dithiotreitol; EA, ethyl acetate; PE, petroleum ether; PMP, para-methoxyphenol; PDI, polydispersity index; PMIRRAS, phase modulation infrared reflection absorption spectroscopy; NMR, nuclear magnetic resonance; CG, coarse grained; FF, force field; NVT, constant number of particles, volume, and temperature; NPT, constant number of particles, pressure, and temperature; AA, all-atoms; DLS, dynamic light scattering; NTA, nanoparticle tracking analysis; EM, electron microscopy; EPR, enhanced permeability and retention factor; PL, phospholipids.

\section{REFERENCES}

(1) Dolmans, D. E. J. G. J., Fukumura, D., and Jain, R. K. (2003) Photodynamic Therapy for Cancer. Nat. Rev. Cancer 3 (5), 380-387.

(2) Cardoso, D., Libardi, S., and Skibsted, L. (2012) Riboflavin as a Photosensitizer. Effects on Human Health and Food Quality. Food Funct. 3, 487-502.

(3) Suwannasom, N., Kao, I., Pruß, A., Georgieva, R., and Bäumler, H. (2020) Riboflavin: The Health Benefits of a Forgotten Natural Vitamin. Int. J. Mol. Sci. 21 (3), 950.

(4) Marschner, S., and Goodrich, R. (2011) Pathogen Reduction Technology Treatment of Platelets, Plasma and Whole Blood Using Riboflavin and UV Light. Transfus Med. Hemoth 38 (1), 8-18.

(5) Wollensak, G., Spoerl, E., and Seiler, T. (2003) Riboflavin/ Ultraviolet-a-Induced Collagen Crosslinking for the Treatment of Keratoconus. Am. J. Ophthalmol. 135 (5), 620-627.

(6) Raiskup, F., Theuring, A., Pillunat, L. E., and Spoerl, E. (2015) Corneal Collagen Crosslinking with Riboflavin and Ultraviolet-A Light in Progressive Keratoconus: Ten-Year Results. J. Cataract Refractive Surg. 41 (1), 41-46.

(7) Darguzyte, M., Drude, N., Lammers, T., and Kiessling, F. (2020) Riboflavin-Targeted Drug Delivery. Cancers 12 (2), 295.

(8) Jayapaul, J., Arns, S., Lederle, W., Lammers, T., Comba, P., Gätjens, J., and Kiessling, F. (2012) Riboflavin Carrier ProteinTargeted Fluorescent USPIO for the Assessment of Vascular Metabolism in Tumors. Biomaterials 33 (34), 8822-8829.
(9) Mertens, M. E., Frese, J., Bölükbas, D. A., Hrdlicka, L., Golombek, S., Koch, S., Mela, P., Jockenhövel, S., Kiessling, F., and Lammers, T. (2014) FMN-Coated Fluorescent USPIO for Cell Labeling and Non-Invasive MR Imaging in Tissue Engineering. Theranostics 4 (10), 1002-1013.

(10) Beztsinna, N., Tsvetkova, Y., Bartneck, M., Lammers, T., Kiessling, F., and Bestel, I. (2016) Amphiphilic Phospholipid-Based Riboflavin Derivatives for Tumor Targeting Nanomedicines. Bioconjugate Chem. 27 (9), 2048-2061.

(11) Beztsinna, N., Solé, M., Taib, N., and Bestel, I. (2016) Bioengineered Riboflavin in Nanotechnology. Biomaterials 80, 121133.

(12) Witte, A. B., Timmer, C. M., Gam, J. J., Choi, S. K., Banaszak Holl, M. M., Orr, B. G., Baker, J. R., and Sinniah, K. (2012) Biophysical Characterization of a Riboflavin-Conjugated Dendrimer Platform for Targeted Drug Delivery. Biomacromolecules 13 (2), 507516

(13) Witte, A. B., Leistra, A. N., Wong, P. T., Bharathi, S., Refior, K., Smith, P., Kaso, O., Sinniah, K., and Choi, S. K. (2014) Atomic Force Microscopy Probing of Receptor-Nanoparticle Interactions for Riboflavin Receptor Targeted Gold-Dendrimer Nanocomposites. J. Phys. Chem. B 118 (11), 2872-2882.

(14) Iida, H., Iwahana, S., Mizoguchi, T., and Yashima, E. (2012) Main-Chain Optically Active Riboflavin Polymer for Asymmetric Catalysis and Its Vapochromic Behavior. J. Am. Chem. Soc. 134 (36), 15103-15113.

(15) Jayapaul, J., Hodenius, M., Arns, S., Lederle, W., Lammers, T., Comba, P., Kiessling, F., and Gaetjens, J. (2011) FMN-Coated Fluorescent Iron Oxide Nanoparticles for RCP-Mediated Targeting and Labeling of Metabolically Active Cancer and Endothelial Cells. Biomaterials 32 (25), 5863-5871.

(16) Ju, S.-Y., and Papadimitrakopoulos, F. (2008) Synthesis and Redox Behavior of Flavin Mononucleotide-Functionalized SingleWalled Carbon Nanotubes. J. Am. Chem. Soc. 130 (2), 655-664.

(17) Massiot, J., Rosilio, V., Ibrahim, N., Yamamoto, A., Nicolas, V., Konovalov, O., Tanaka, M., and Makky, A. (2018) Newly Synthesized Lipid-Porphyrin Conjugates: Evaluation of Their Self-Assembling Properties, Their Miscibility with Phospholipids and Their Photodynamic Activity In Vitro. Chem. - Eur. J. 24 (72), 19179-19194.

(18) Huynh, E., and Zheng, G. (2014) Porphysome Nanotechnology: A Paradigm Shift in Lipid-Based Supramolecular Structures. Nano Today 9 (2), 212-222.

(19) Komatsu, T., Moritake, M., Nakagawa, A., and Tsuchida, E. (2002) Self-Organized Lipid-Porphyrin Bilayer Membranes in Vesicular Form: Nanostructure, Photophysical Properties, and Dioxygen Coordination. Chem. - Eur. J. 8 (23), 5469-5480.

(20) Lovell, J. F., Jin, C. S., Huynh, E., Jin, H., Kim, C., Rubinstein, J. L., Chan, W. C. W., Cao, W., Wang, L. V., and Zheng, G. (2011) Porphysome Nanovesicles Generated by Porphyrin Bilayers for Use as Multimodal Biophotonic Contrast Agents. Nat. Mater. 10 (4), 324332.

(21) Yeh, M.-K., Hsin-I Chang, and Ming-Yen Cheng (2011) Clinical Development of Liposome-Based Drugs: Formulation, Characterization, and Therapeutic Efficacy. Int. J. Nanomed. 7, 49-60.

(22) Vermeer, L. S., de Groot, B. L., Réat, V., Milon, A., and Czaplicki, J. (2007) Acyl Chain Order Parameter Profiles in Phospholipid Bilayers: Computation from Molecular Dynamics Simulations and Comparison with $2 \mathrm{H}$ NMR Experiments. Eur. Biophys. J. 36 (8), 919-931.

(23) Ingólfsson, H. I., Lopez, C. A., Uusitalo, J. J., de Jong, D. H., Gopal, S. M., Periole, X., and Marrink, S. J. (2014) The Power of Coarse Graining in Biomolecular Simulations. WIREs Comput. Mol. Sci. 4 (3), 225-248.

(24) Kmiecik, S., Gront, D., Kolinski, M., Wieteska, L., Dawid, A. E., and Kolinski, A. (2016) Coarse-Grained Protein Models and Their Applications. Chem. Rev. 116 (14), 7898-7936.

(25) Papoian, G. A. (2017) Coarse-Grained Modeling of Biomolecules, CRC Press, Boca Raton, FL. 
(26) Singh, N., and Li, W. (2019) Recent Advances in CoarseGrained Models for Biomolecules and Their Applications. Int. J. Mol. Sci. 20 (15), 3774-3795.

(27) Marrink, S. J., de Vries, A. H., and Mark, A. E. (2004) Coarse Grained Model for Semiquantitative Lipid Simulations. J. Phys. Chem. B 108 (2), 750-760.

(28) Periole, X., and Marrink, S.-J. (2013) The Martini CoarseGrained Force Field. In Biomolecular Simulations: Methods and Protocols (Monticelli, L., and Salonen, E., Eds.) pp 533-565, Humana Press, Totowa, NJ.

(29) Marrink, S. J., Risselada, H. J., Yefimov, S., Tieleman, D. P., and de Vries, A. H. (2007) The MARTINI Force Field: Coarse Grained Model for Biomolecular Simulations. J. Phys. Chem. B 111 (27), $7812-7824$

(30) Martini: Coarse Grain Forcefield for Biomolecules. http:// cgmartini.nl/.

(31) Humphrey, W., Dalke, A., and Schulten, K. (1996) VMD: Visual Molecular Dynamics. J. Mol. Graphics 14 (1), 33-38.

(32) Yoneda, F., Sakuma, Y., Ichiba, M., and Shinomura, K. (1976) Syntheses of Isoalloxazines and Isoalloxazine 5-Oxides. A New Synthesis of Riboflavin. J. Am. Chem. Soc. 98 (3), 830-835.

(33) Frier, C., Décout, J.-L., and Fontecave, M. (1997) Method for Preparing New Flavin Derivatives: Synthesis of Flavin-Thymine Nucleotides and Flavin-Oligonucleotide Adducts. J. Org. Chem. 62 (11), 3520-3528.

(34) Iwashita, M., Makide, K., Nonomura, T., Misumi, Y., Otani, Y., Ishida, M., Taguchi, R., Tsujimoto, M., Aoki, J., Arai, H., et al. (2009) Synthesis and Evaluation of Lysophosphatidylserine Analogues as Inducers of Mast Cell Degranulation. Potent Activities of Lysophosphatidylthreonine and Its 2-Deoxy Derivative. J. Med. Chem. 52 (19), 5837-5863.

(35) Liang, J.-Y., Yuann, J.-M. P., Hsie, Z.-J., Huang, S.-T., and Chen, C.-C. (2017) Blue Light Induced Free Radicals from Riboflavin in Degradation of Crystal Violet by Microbial Viability Evaluation. J. Photochem. Photobiol., B 174, 355-363.

(36) Insińska-Rak, M., and Sikorski, M. (2014) Riboflavin Interactions with Oxygen-A Survey from the Photochemical Perspective. Chem. - Eur. J. 20 (47), 15280-15291.

(37) Vasilaki, A. T., McMillan, D. C., Kinsella, J., Duncan, A., O'Reilly, D. S. J., and Talwar, D. (2010) Relation between Riboflavin, Flavin Mononucleotide and Flavin Adenine Dinucleotide Concentrations in Plasma and Red Cells in Patients with Critical Illness. Clin. Chim. Acta 411 (21), 1750-1755.

(38) Van der Spoel, D., Lindahl, E., Hess, B., Groenhof, G., Mark, A. E., and Berendsen, H. J. C. (2005) GROMACS: Fast, Flexible, and Free. J. Comput. Chem. 26 (16), 1701-1718.

(39) Parrinello, M., and Rahman, A. (1981) Polymorphic Transitions in Single Crystals: A New Molecular Dynamics Method. J. Appl. Phys. 52 (12), 7182-7190.

(40) Berendsen, H. J. C., Postma, J. P. M., van Gunsteren, W. F., DiNola, A., and Haak, J. R. (1984) Molecular Dynamics with Coupling to an External Bath. J. Chem. Phys. 81 (8), 3684-3690.

(41) Wassenaar, T. A., Pluhackova, K., Böckmann, R. A., Marrink, S. J., and Tieleman, D. P. (2014) Going Backward: A Flexible Geometric Approach to Reverse Transformation from Coarse Grained to Atomistic Models. J. Chem. Theory Comput. 10 (2), 676-690.

(42) Bangham, A. D., Standish, M. M., and Watkins, J. C. (1965) Diffusion of Univalent Ions across the Lamellae of Swollen Phospholipids. J. Mol. Biol. 13 (1), 238-252.

(43) Blaudez, D., Buffeteau, T., Cornut, J. C., Desbat, B., Escafre, N., Pezolet, M., and Turlet, J. M. (1994) Polarization Modulation FTIR Spectroscopy at the Air-Water Interface. Thin Solid Films 242 (1), $146-150$.

(44) Schneider, C. A., Rasband, W. S., and Eliceiri, K. W. (2012) NIH Image to ImageJ: 25 Years of Image Analysis. Nat. Methods 9 (7), 671-675. 